\title{
Kernos
}

Revue internationale et pluridisciplinaire de religion grecque antique

$25 \mid 2012$

Varia

\section{Giulia PEDRUCCI, Cibele Frigia e la Sicilia. I santuari rupestri nel culto delle dea}

\section{Paolo Daniele Scirpo}

(2) OpenEdition

Journals

\section{Edizione digitale}

URL: http://journals.openedition.org/kernos/2064

DOI: 10.4000/kernos.2064

ISSN: 2034-7871

\section{Editore}

Centre international d'étude de la religion grecque antique

\section{Edizione cartacea}

Data di pubblicazione: 26 octobre 2012

Paginazione: 360-362

ISSN: 0776-3824

Notizia bibliografica digitale

Paolo Daniele Scirpo, "Giulia PEDRuccl, Cibele Frigia e la Sicilia. I santuari rupestri nel culto delle dea », Kernos [En ligne], 25 | 2012, mis en ligne le 01 octobre 2012, consulté le 21 septembre 2020. URL: http://journals.openedition.org/kernos/2064 ; DOI : https://doi.org/10.4000/kernos.2064 


\section{Giulia PEDRUCCI, Cibele Frigia e la Sicilia. I santuari rupestri nel culto delle dea}

Paolo Daniele Scirpo

\section{NOTIZIA}

Giulia PEDRUCCI, Cibele Frigia e la Sicilia. I santuari rupestri nel culto delle dea, Roma, «L'Erma» di Bretschneider, 2009. 1 vol. 17,5 × 24,5 cm, 156 p., 21 pl. (Studia Archaeologica, 168). ISBN : 978-88-8265-560-0.

1 Sotto il patrocinio dell'Istituto di Studi Acrensi (I.S.A.) di Palazzolo Acreide, del locale Rotary Club e del Comune ibleo, esce per le edizioni dell'«Erma» di Bretschneider la monografia dedicata alla dea Cibele, frutto di un lavoro che l'A. porta avanti da anni e divisa in tre sezioni di diseguale lunghezza. Nella prima parte (p.11-36), attraverso un'attenta indagine storico-religiosa, l'A. prova a rispondere all'annoso quesito: "Chi è Cibele?" L'analisi della questione onomastica che ruota attorno all'assenza di un nome

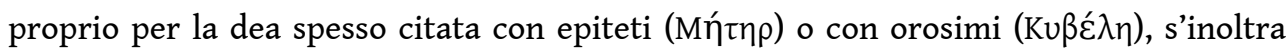
nella densa coltre che avvolge la nascita stessa della divinità, frutto di una sorta di "ingegneria politeistica". La sua stessa iconografia mai definitivamente stabilita, fu sempre in bilico fra una singola raffigurazione ed una "doppia" e testimonierebbe il lungo processo di ellenizzazione che la dea frigia subì probabilmente in un ambiente fertile e ricettivo quale quello costituito dalle poleis microasiatiche, laddove cioè, secondo l'A., avvenne il primo contatto.

Nella seconda parte del volume (p. 37-72) sono esposte tutte le tracce materiali della presenza della Dea in Sicilia. Le poche fonti epigrafiche e gli accenni tratti da quelle letterarie non possono che far da contorno all'unico (al momento) vero santuario metroaco noto sull'isola: quello di Akrai. Il primo incontro dell'A. con la dea è avvenuto in un articolo ${ }^{1}$ dove, prendendo in esame una parte un po' tralasciata del santuario 
acrense, cioè i due spiazzi con altari circolari ed i gradini scolpiti nella roccia, l'A. giunse a conclusioni che scalfivano la monolitica ricostruzione di Bernabò Brea ${ }^{2}$, ribadita e puntualizzata da Sfameni Gasparro ${ }^{3}$, della provenienza anatolica dell'ispirazione del santuario. Si evidenziò così che il legame strettissimo della dea con la roccia, di ascendenza hittita, parve confermare il possibile itinerario compiuto dalla dea da Oriente a Occidente, già intravisto da Guarducci ${ }^{4}$. Nei suoi successivi lavori, l'A. postulò un legame della dea anche con l'area elima ${ }^{5}$ e ribadì le ascendenze siriane della dea Kubaba ${ }^{6}$. Tipologia e iconografia infatti, legherebbero il santuario acrense a quelli della Frigia, di datazione però molto più antica.

3 La terza parte (p. 73-130), invece, ci riporta in Anatolia dove la dea sarebbe nata nel II millennio a.C., e dove si sarebbe gradualmente fatta strada nelle credenze religiose degli Ittiti, e tramite loro giungere in Frigia ed infine, in Lidia. L'A. passa così in rassegna i monumenti rupestri frigi d'età arcaica e classica e, dopo una breve parentesi nella quale ipotizza un legame fra la Sicilia e l'Anatolia ritornando sulle particolarità del santuario acrense sopra accennate, termina il capitolo con l'accurato esame dei monumenti rupestri di età ellenistica. Ai tanti interrogativi che la figura di Cibele porta ancor oggi con sé, l'A.tenta di dare delle risposte plausibili e, per certi versi, auspicabili. Data l'antichità dei santuari frigi e la coeva fioritura delle poleis micrasiatiche, frutto della "prima colonizzazione" della prima età del Ferro, appare evidente che in un momento qualsiasi la religiosità delle popolazione autoctone si sia incontrata con quella delle philai elleniche, ancora magmaticamente protese ad "assimilare" concezioni religiose non patrie. Per quanto riguarda il secondo quesito, ovvero come il culto della dea sia arrivato in Sicilia, già Guarducci sulla base del coccio di Locri Epizefiri, datato alla fine del VII secolo a.C., propose una rotta che partendo dagli altipiani della Frigia, con la mediazione dei coloni di Colofone e Siris, toccasse il suolo calabro a Locri e grazie agli ottimi rapporti intercorsi nei secoli fra le due apoikiai doriche, giungesse a Siracusa (e da lì ad Akrai). L'A. ne sposa in parte la tesi senza però mancare di evidenziare l'intensa componente asiatica che si mantenne nelle raffigurazioni acrensi. La forte valenza ctonia nella Cibele venerata in Sicilia però, farebbe propendere l'A. per un suo arrivo in età più antica, pre-greca, quando essa entrò probabilmente in contatto con divinità autoctoni (elime?) con caratteristiche a lei simili. Questa mescolanza avrebbe pertanto permesso il perdurare di certi aspetti originari della dea che nel resto del mondo greco andarono col tempo perduti (p. 132). Oltre agli immancabili e fastidiosi refusi tipografici, certe assenze nella pur vasta bibliografia consultata (p. 139-156) mostrano il fianco a critiche in alcuni punti dedicati soprattutto al santuario acrense, senza però inficiare del tutto le conclusioni generali della ricerca.

4 L'A. porta per mano il lettore in un viaggio spazio-temporale enorme dagli altipiani dell'Anatolia alle valli della Sumeria, passando per Ebla, Karkemish e la Cilicia, senza purtroppo fornire una semplice cartina geografica dove indicare le tappe di questa sua indagine. Inoltre l'ambigua citazione della toponomastica locale che oscilla da quella autoctona, a quella greca e persino turca, non facilita affatto il compito di chi si avvicina a temi delle antiche civiltà orientali (ittita, frigia, lidia, hurrita, eblaita, etc.). Con un apparato fotografico in generale di buona qualità, la monografia, per quanto riguarda il santuario di Akrai, ha come pregio-limite, quello di ripubblicare le splendide fotografie volute da Bernabò Brea ed i disegni di Rosario Carta, senza aggiungere nulla di nuovo, nemmeno un'immagine dello stato attuale (scandalosamente pietoso) del monumento. Da non attribuirsi certamente all'A. è l'errore nella tavola XII dove, invece 
della latomia (i c.d. «Templi Ferali») posta sulle pendici sud-est della città, è raffigurata quella urbana dell'Intagliatella con in primo piano il grande rilievo rupestre, a carattere sacro ${ }^{7}$.

5 Pur coi i suoi fisiologici limiti e con le mille questioni ancora aperte sulla provenienza orientale della dea frigia, lo studio di G.P. rappresenta un ottimo punto di partenza, sintetico e accessibile, sull'argomento. Si accoglierebbe di certo con favore una seconda edizione, aggiornata ed ampliata, che possa stare così accanto degnamente alle più fondamentali (e non molte) monografie dedicate alla Grande Madre degli Dei.

\section{NOTE}

1. G. PEDRUCCI, «I santuari rupestri metroaci fra Sicilia e Anatolia », RSA 35 (2005), p. 165-179.

2. L. BERNABò BREA, Akrai, Catania, 1956, p. 89-113.

3. G. SFAMENI GASPARRO, I culti orientali in Sicilia, Leiden, 1973, p. 148-149.

4. M. GUARDUCCI, «Cibele in un'epigrafe arcaica di Locri Epizefiri», in Klio 52 (1970), p. 133-138.

5. G. PEDRUCCI, «Presenze Metroache in area elima?», Hormos 9 (2007), p. 437-441.

6. G. PEDRUCCI, «Kubaba: presenze anatoliche e antecedenti siriani», Vicino Oriente 14 (2008), p. 161-179.

7. P.D. SCIRPO, «The Founder-Cult of Hieron II at Akrai: The Rock-Relief from Intagliatella's Latomy», in S.A. PAIPETIS, M. CECCARELLI (eds), The Genius of Archimedes - 23 Centuries of Influence on Mathematics, Science, and Engineering, Dordrecht, Springer, 2010 (History of Mechanism and Machine Science, 11), p. 411-420.

\section{AUTORI}

\section{PAOLO DANIELE SCIRPO}

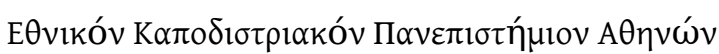

